\title{
Antibacterial properties of glycosylated surfaces: variation of the glucosidal moiety and fatty acid conformation of grafted microbial glycolipids
}

Claire Valotteau ${ }^{1,2}$, Sophie Roelants ${ }^{3,4}$, Prabhu Dasaiyan ${ }^{5}$, Susanne Zibek ${ }^{6}$, Michael Günther ${ }^{7}$, Wim Soetaert ${ }^{3,4}$, Bernd Everaert ${ }^{4}$, Claire-Marie Pradier ${ }^{1}$, Florence Babonneau ${ }^{2}$, Niki Baccile ${ }^{2, \$}$, Vincent Humblot ${ }^{1,8, \$}$.

${ }^{1}$ Sorbonne Université, Laboratoire de Réactivité de Surface (LRS), UMR CNRS 7197, 4 place Jussieu, Paris, F-75005, France.

${ }^{2}$ Sorbonne Université, Laboratoire de Chimie de la Matière Condensée de Paris (LCMCP), UMR CNRS 7574, 4 place Jussieu, Paris, F-75005, France.

${ }^{3}$ Ghent University, Centre for Industrial Biotechnology and Biocatalysis (InBio.be), Coupure Links 653, B-9000 Gent, Belgium

${ }^{4}$ Bio Base Europe Pilot Plant, Rodenhuizekaai 1, B-9000 Gent, Belgium

${ }^{5}$ Physical and Materials Chemistry Division, CSIR - National Chemical Laboratory, Pune - 411008 , India

${ }^{6}$ Fraunhofer Institute for Interfacial Engineering and Biotechnology IGB, Nobelstraße 12, D-70569 Stuttgart, Germany

${ }^{7}$ Universität Stuttgart, Institut für Grenzflächenverfahrenstechnik, Nobelstraße 12, D-70569 Stuttgart, Germany

${ }^{8}$ FEMTO-ST Institute, Université Bourgogne Franche-Comté, UMR CNRS 6174, 15B avenue des Montboucons, 25030 Besancon Cedex, France

${ }^{\$}$ Corresponding authors: niki.baccile@ sorbonne-universite.fr; vincent.humblot@femto-st.fr 


\begin{abstract}
Glycosylated surfaces can display antimicrobial properties. It has been shown that sophorolipids can be used to develop biocidal coatings against Gram-positive and Gramnegative bacteria, but with a limited efficiency so far. Therefore, it appears necessary to further investigate the surface antibacterial activity of a broader set of structurally related glycolipids. The present work explores the influence of the glucosidic moiety (gluco-, sophoro-, cellobio-) and the fatty acid backbone (saturated, cis or trans monounsaturated). We show that the fatty acid backbone plays an important role: cis derivative of sophorolipids (SL) grafted onto model gold surfaces has better biocidal properties than saturated $\left(\mathrm{SL}_{0}\right)$ and trans monounsaturated $\left(\mathrm{SL}_{\mathrm{t}}\right)$ molecules, which appear to be inefficient. The number of glucose units is also a key factor: a one-third decrease in antibacterial activity is observed when having one glucose unit (GL) compared to two (SL).Sugar acetylation ( $\mathrm{SL}_{\mathrm{a}}$ ) does not seem to have an impact on the biocidal properties of surfaces. These results are not limited to sophorolipids, cellobioselipids (CL) leading to similar antibacterial observations.
\end{abstract}




\section{INTRODUCTION}

Microbial attachment to surfaces and the subsequent biofilm development can lead to material deterioration $^{1}$ and health issues ${ }^{2}$. Established biofilms are difficult to eradicate and their treatments are costly and ineffective. Therefore, many studies aimed at developing preventive antimicrobial coatings on surfaces. Antibiotics, chloride and quaternary ammonium compounds (CAQs), incorporated into or grafted onto (polymeric) matrices are commonly used. ${ }^{3-5}$ However, these strategies are nowadays criticized because of their ecotoxicity and the increased bacterial resistance to these chemicals, which highlights the need for alternative eco-responsible approaches $^{6,7}$, with for instance less toxic QACs as shown recently by Forman et al. ${ }^{8}$ Biosurfactants, such as free fatty acids, glycolipides or monoglycerides, are part of a class of molecules obtained from the fermentation of vegetable oils and sugar, appear to be promising ecofriendly and affordable candidates. ${ }^{9-12}$

Microbial biosurfactants are biobased compounds obtained by the fermentation process of sugars and lipids, and their antimicrobial properties have been long described. ${ }^{13,}{ }^{14}$ Several reviews have been published on this topic since more than a decade, ${ }^{15,16}$ while a number of recent studies has shown additional interesting properties like adjuvant effects with other antimicrobial compounds ${ }^{17-19}$ or anti-biofilm properties. ${ }^{20-22}$ On this basis, specific formulation in the field of, e.g., oral hygiene, were recently developed. ${ }^{17,23}$ The anti-adhesive properties of biosurfactants against pathogenic organisms like E. faecalis, S. mutans or E. coli are particularly interesting, ${ }^{16}$ although the general protocol to study anti-adhesion and anti-biofilm properties of biosurfactants only consider a surface dispersion of the compound, this making it particularly vulnerable to washing. To address this issue, sophorolipids were immobilized in surfaces and they have shown a short-term antiadhesive effect (within the first seconds of contact), ${ }^{24}$ probably due to repulsive short-distance hydration forces, but also a long-term (> 1h) biocidal effect. These antimicrobial effects are characterized by lysis of both Gram-positive and Gramnegative bacterial cells and decreased bacterial growth capacities. ${ }^{25}$ However, only up to 50\% of bacterial cells are affected, whereas much higher efficiency is expected from antimicrobial surfaces. Therefore, it seems necessary to further investigate the potential of glycosylated surfaces to improve their antimicrobial activity and to understand the effect of carbohydrates.

It has already been demonstrated that no biocidal activity can be detected on surfaces grafted solely with the aliphatic backbone of sophorolipids, nor with sophorolipids having a fully saturated aliphatic chain. ${ }^{26}$ Thus, the antimicrobial properties of surfaces functionalized 
with sophorolipids are attributed to the presence of the carbohydrate moiety ${ }^{26}$ and correlated to the configuration of the aliphatic chain ${ }^{26}$, as well as to the surface density of sophorolipids ${ }^{25}$. Besides, replacing the glucose by several sugars, such as fructose, xylose, ribose, lactose, mannose, arabinose, galactose, in the fermentation medium used for the production of the sophorolipids induces variations in the carbohydrate moieties that further influence the antimicrobial activities of the resulting compounds. ${ }^{27}$ More generally, the properties of glycosylated surfaces, known as glycoarrays, developed to study carbohydrate-binding proteins and carbohydrate-processing enzymes, ${ }^{28}$ are influenced by carbohydrates conformation ${ }^{29}$ and the biochemical affinities of carbohydrates after immobilization on a surface can differ from those in solution ${ }^{30}$. These observations encouraged us to explore a broader molecular portfolio and to seek a better understanding of the possible molecular parameters underlying the antimicrobial effect of glycosylated surfaces. Building on the methodology and protocols previously developed for a specific glycolipid ${ }^{26}$, this work investigates rational variations in the glycosidic headgroup and chain configuration (Figure 1). The typical disaccharide sophorose is replaced by an acetylated sophorose (acetylated sophorolipid, $\mathrm{SL}_{\mathrm{a}}$, known to be more resistant to enzymatic degradation ${ }^{31}$ and more active in solution ${ }^{32}$ ), or a monosaccharide (glucolipid, GL, that could be residue of the sophorolipids degradation by bacterial glycosidases ${ }^{33,34}$ ). An alternative linkage of sophorose to the fatty acid chain is also being considered (branched sophorolipid, $\mathrm{SL}_{\mathrm{b}}$ ). The lipidic structure is varied from cis (oleic acid sophorolipid, SL) to trans (elaidic acid sophorolipid, $\mathrm{SL}_{\mathrm{t}}$ ) and saturated (stearic acid sophorolipid, $\mathrm{SL}_{0}$ ) configurations. Cellobioselipid (CL), another microbial glycolipid with a cellobiose headgroup, produced by the fungus Ustilago maydis and also known to be a membrane disruptor, ${ }^{35}$ is also tested for its structural proximity to sophorolipids. All compounds were immobilized on gold surfaces by grafting their free carboxylic acid moiety onto a self-assembled monolayer of short thiol amines, following the protocols developed in our previous works. ${ }^{25,26}$ The thickness, composition and homogeneity of these substrates were characterized by Polarization Modulation Reflection Absorption Infrared Spectroscopy (PM-RAIRS) and X-ray photoelectron spectroscopy (XPS), followed by evaluation of the adhesion, membrane integrity and cultivability of the non-pathogenic Gram-positive bacterium Listeria ivanovii. We found that the surface antimicrobial properties appear to be strongly affected by carbohydrate configuration and accessibility: for sophorolipid analogues with the same molecular surface density, it appears that the monounsaturated cis bond is necessary to enhance antibacterial activity. In addition, the nature of the sugar part, in particular the number and acetylation extent of glucose units, influences also the biocidal efficiency. 


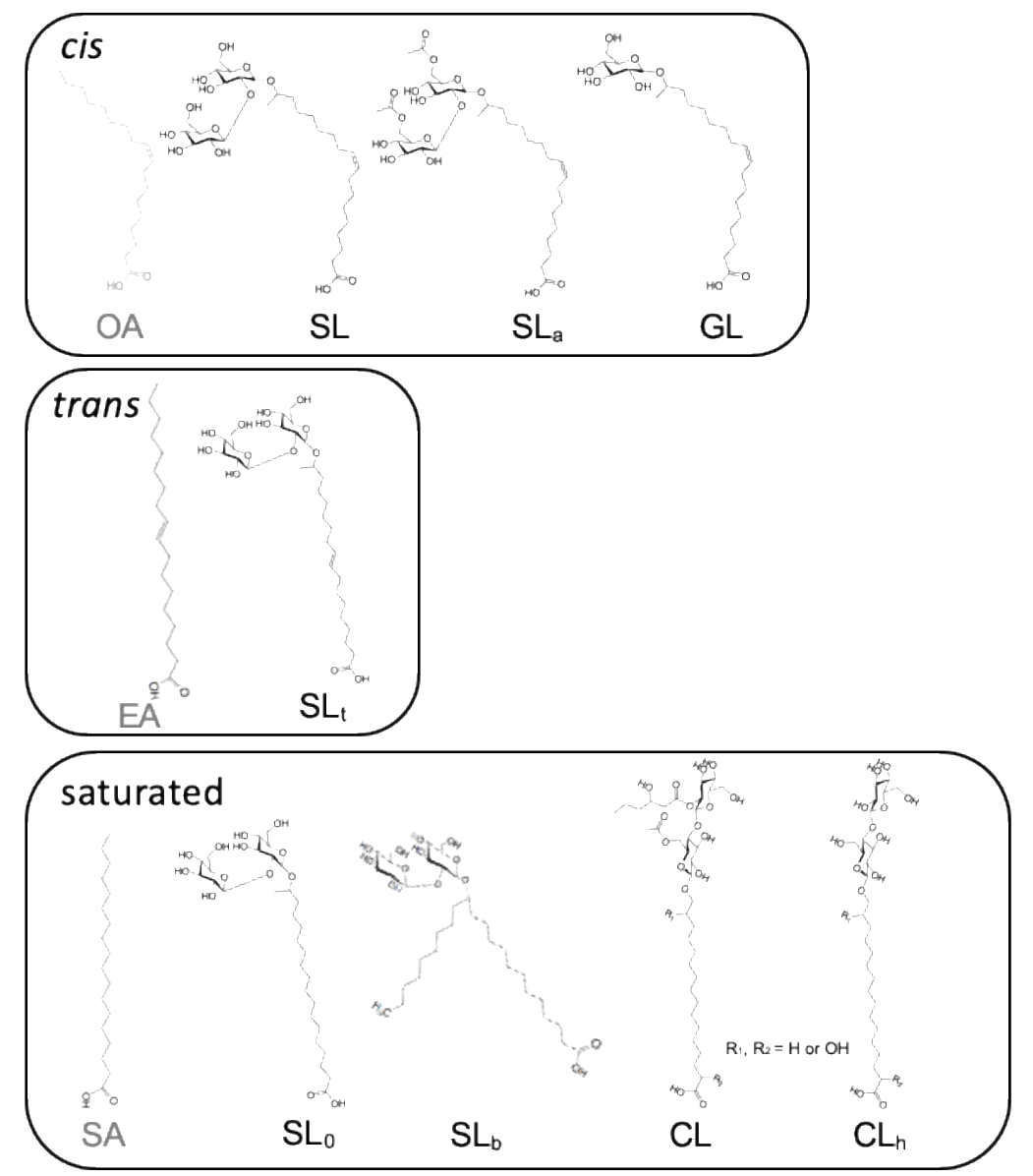

Figure 1. Set of microbial glycolipids presenting rational variation of the glycosidic headgroup (horizontally) and chain configuration (vertically).

The different acid chains are shown in grey as references.

3 glycolipids have a cis $\mathrm{C} 18$ oleic acid (OA) chain: the typical sophorolipid (SL), the acetylated sophorolipid (SLa) and the glucolipid (GL).

The trans sophorolipid ( $\mathrm{SL}_{\mathrm{t}}$ ) has a trans $\mathrm{C} 18$ elaidic acid (EA) chain.

The last glycolipids have a fully saturated chain: the saturated stearic acid (SA) sophorolipid $\left(\mathrm{SL}_{0}\right)$, the branched sophorolipid $\left(\mathrm{SL}_{b}\right)$, the cellobioselipid $(\mathrm{CL})$ and the hydrolysed cellobioselipid $\left(\mathrm{CL}_{h}\right)$. For the latter, $\mathrm{R} 1=\mathrm{H}$ or $\mathrm{OH}$ and $\mathrm{R} 2=\mathrm{H}$ or $\mathrm{OH}$, as described in the materials and method section.

\section{MATERIALS AND METHODS}

Cysteamine (cys), N-hydroxysuccinimide (NHS),1-(3-(dimethylamino)propyl)-Nethylcarbodiimide hydrochloride (EDC), oleic acid (OA), stearic acid (SA) and elaidic acid (EA) were obtained from Sigma-Aldrich (Saint Quentin Fallavier, France). All solvents were 
reagent-grade and were used without any further purification. Water was purified with a MilliQ system (Millipore, resistivity $>18 \mathrm{M} \Omega \mathrm{cm}^{-1}$ ) from EMD Millipore Corp. (Billerica, MA). Glass substrates, coated successively with a $50 \AA$ thick layer of chromium and a $200 \mathrm{~nm}$ thick layer of gold, were purchased from Arrandee (Werther, Germany).

All glycolipids (see Figure 1 for scheme and formula) used in this work have been used from previous studies, to which one should refer to for their characterization.

Deacetylated acidic cis C18 sophorolipids (SL) were obtained by a classical alkaline hydrolysis treatment of a commercial acidic and lactone mixture of sophorolipids purchased from Soliance (France) (Sopholiance S; batch number, 11103A; dry content, $60 \pm 6 \%$ ). ${ }^{26,36}$

The sophorolipid compound $\mathrm{SL}_{0}$ constituted by a fully saturated $\mathrm{C} 18$ fatty acid (stearic acid, SA) was derived from the previous one using a Pd-catalyzed hydrogenation reaction described in ref ${ }^{26,37}$

Acetylated acidic cis $\mathrm{C} 18$ sophorolipids $\left(\mathrm{SL}_{\mathrm{a}}\right)$ have been produced using a Starmerella bombicola lactone esterase knock out strain $(\Delta s b l e) .{ }^{38}$ Their synthesis and composition has been described in detail in ref. ${ }^{39}$ and they were kindly provided by the Bio Base Europe Pilot Plant (Ghent, Belgium) and used as such.

Deacetylated acidic cis C18 glucolipids (GL) were produced by the $S$. bombicola strain $\Delta u g t B 1^{40}$ and provided by the Center for Industrial Biotechnology and Biocatalysis of Ghent University. Their synthesis and purification process has been described in ref. ${ }^{41}$.

Acidic sophorolipids bearing a trans $\mathrm{C} 18$ fatty acid (elaidic acid, EA), $\mathrm{SL}_{\mathrm{t}}$, have also been employed. $\mathrm{SL}_{\mathrm{t}}$, produced at the National Chemical Laboratory at Pune (India), is obtained by the fermentation process by $S$. bombicola using elaidic acid as carbon support. Their synthesis and purification procedure is given elsewhere. ${ }^{42}$

The compound $\mathrm{SL}_{b}$ refers to a deacetylated acidic sophorolipid bearing a glycosidic bond with the $\mathrm{C} 13$ position of 13-hydroxydocosanoic acid. For its peculiar morphology, it is named "branched sophorolipid". The compound used in this work was recently produced ${ }^{43}$ according to a published procedure ${ }^{44}$ by the yeast Rhodotorula bogoriensis MUCL 11796, recently renamed Pseudohyphozyma bogoriensis.

Cellobioselipids (CL) were provided by the Industrial Biotechnology group of Fraunhofer Institute for Interfacial Engineering and Biotechnology (Stuttgart). They are produced by the fungus Ustilago maydis ${ }^{45}$ as a mixture of hydroxyled and non-hydroxyled congeners. The hydrolyzed cellobioselipids $\left(\mathrm{CL}_{h}\right)$ were obtained by alkaline hydrolysis and further acidification at neutral $\mathrm{pH}$. Alkaline hydrolysis and characterization of both CL and $\mathrm{CL}_{h}$ are described in ref. ${ }^{41}$.Both CL and CLH are mixtures of structurally similar congeners where 
both $\mathrm{R} 1=\mathrm{H}$ or $\mathrm{OH}$ and $\mathrm{R} 2=\mathrm{H}$ or $\mathrm{OH}$ and where the proportion of $\mathrm{R} 1=\mathrm{H}$ was evaluated to about $40 \%$ by ${ }^{1} \mathrm{H}$ NMR. ${ }^{41}$.

\section{Surface preparation and characterization.}

\section{Glycosylated surfaces}

All glycolipids were grafted onto flat gold substrates via a self-assembled monolayer of short aminothiols, as described previously. ${ }^{25,26}$ Briefly, the substrates were first annealed in a butane flame to ensure a good crystallinity of the uppermost layer and afterwards cleaned by UV-ozone during $15 \mathrm{~min}$. Substrates were then immersed in an ethanolic solution of cysteamine (cys) at $10 \mathrm{mM}$ for $3 \mathrm{~h}$, sonicated in ethanol for $5 \mathrm{~min}$ and then rinsed successively in ethanol and in ultrapure water before being dried under a flow of dried air. The samples were then immersed for $3 \mathrm{~h}$ into solutions of $8 \mathrm{mM}$ glycolipids (equivalent to $50 \mathrm{mg} / \mathrm{mL}$ for SL) ${ }^{25}$; the terminal carboxylic acid having been previously activated into a succinimide ester using a mixture of EDC and NHS (molar ratio NHS:EDC=1:2). Finally, after incubation, sonication and successive rinsing in ultrapure water and ethanol were carried out to remove non-covalently grafted reactants before drying under a flow of dried air. All samples were characterized by Polarization Modulation Reflection Absorption Infrared Spectroscopy (PM-RAIRS) after each step of functionalization to guarantee the successful grafting of the different primers, fatty acids and glycolipids.

\section{$X$-ray Photoelectron Spectroscopy (XPS)}

XPS analyses were performed using an Omicron Argus X-ray photoelectron spectrometer, equipped with a monochromated $\mathrm{Al} \mathrm{K}_{\alpha}$ radiation source $(h v=1486.6 \mathrm{eV})$ and a $300 \mathrm{~W}$ electron beam power. The emission of photoelectrons from the sample was analyzed at a take-off angle of $90^{\circ}$ under ultra-high vacuum conditions $\left(\leq 10^{-10}\right.$ Torr $)$. Spectra were recorded with a $100 \mathrm{eV}$ pass energy for the survey scan and $20 \mathrm{eV}$ pass energy for the $\mathrm{C} 1 \mathrm{~s}, \mathrm{O} 1 \mathrm{~s}, \mathrm{~N} 1 \mathrm{~s}$, $\mathrm{S} 2 \mathrm{p}$ regions. Binding energies were calibrated against the $\mathrm{Au} 4 \mathrm{f}_{7 / 2}$ binding energy at $84.0 \mathrm{eV}$ and element peak intensities were corrected by Scofield factors. ${ }^{46}$ The peak areas were determined after subtraction of a linear background. The spectra were fitted using Casa XPS v.2.3.15 software (Casa Software Ltd., U.K.) and applying a Gaussian/Lorentzian ratio G/L equal to $70 / 30$.

\section{Polarization Modulation Reflection Absorption Infrared Spectroscopy (PM-RAIRS)}


Infrared (IR) analysis of the surfaces has been done using PM-RAIRS. A Nicolet Nexus 5700 FT-IR spectrometer equipped with a nitrogen-cooled $\mathrm{HgCdTe}$ wide band detector has been employed. Infrared spectra were recorded at $8 \mathrm{~cm}^{-1}$ resolution, by coaddition of 128 scans. A ZnSe grid polarizer and a ZnSe photoelastic modulator were placed prior to the sample in order to modulate the incident beam between $\mathrm{p}$ and s polarizations (HINDS Instruments, PM90, modulation frequency $=36 \mathrm{kHz}$ ). The sum and difference interferograms were processed and underwent Fourier-transformation to yield the PM-RAIRS signal which is the differential reflectivity $\left(\Delta R / R_{0}\right)=\left(R_{p}-R_{s}\right) /\left(R_{p}+R_{s}\right)$ where $R_{p}$ and $R_{s}$ are respectively the $p$ - and $s-$ polarized components of radiation. All measurements (on control and functionalized surfaces) were performed during the same experimental session, thus reducing at minimum intensity variations of the background, beam intensity and alignment.

\section{Antimicrobial activity.}

\section{Strain and culture conditions}

The antibacterial properties of the glycosylated surfaces were tested against the nonhuman pathogenic Gram-positive bacteria Listeria ivanovii Li4pVS2. Bacteria were cultivated overnight in Brain Heart Infusion (BHI) broth (BD Difco, France) at $37^{\circ} \mathrm{C}$ under agitation (250 rpm). The cells from stationary cultures were harvested by centrifugation at 10,000 $\mathrm{g}$ for 5 min and dispersed in an isotonic sterile solution $(\mathrm{NaCl} 0.9 \%)$ in order to obtain an optical density at $620 \mathrm{~nm}$ of 0.05 , which corresponds to approximately $5 \cdot 10^{6}$ colony forming units per $\mathrm{mL}(\mathrm{cfu} / \mathrm{mL})$.

\section{Deposition of bacteria on samples}

All samples were sterilized by immersion in a solution of $70 \%$ ethanol (in water) and dried in a sterile environment. They were then inoculated with a $100 \mu \mathrm{L}$ drop of bacterial suspension and incubated for $3 \mathrm{~h}$ at room temperature in a wet atmosphere. After this time, surfaces were washed five times with $100 \mu \mathrm{L}$ of isotonic sterile solution $(\mathrm{NaCl} 0.9 \%)$ to remove all non-adhering bacteria.

\section{Evaluation of bacteria adhesion by infrared spectroscopy}

The relative amounts of bacteria adsorbed on each substrate were evaluated by considering the IR signal collected by scanning each surface (after drying under sterile laminar air-flow), as described in previous works. ${ }^{25,26}$ 
The attachment of bacteria onto the different surfaces is expressed as a percentage of amide I and II bands area (between $1700 \mathrm{~cm}^{-1}$ and $1500 \mathrm{~cm}^{-1}$ ) compared to control gold substrates: Adhesion $(\%)=100 \times($ area of amide bands on sample $) /($ area of amide bands on gold). The uncertainty attached to this percentage comes from the propagation of the uncertainties attached to the measurement of amide bands area on both the considered surface and the gold substrate. These results were confirmed by repeating the same procedure on three different sets of samples prepared with independent bacterial cultures. 


\section{Evaluation of bacteria damaging by fluorescent staining}

The proportion of damaged bacterial cells was evaluated by counting bacteria after staining with Live/Dead Bacterial Viability Kit (BacLight $\left.{ }^{\circledR}\right)$. After rinsing of inoculated sample, the adhered bacteria were labeled with $10 \mu \mathrm{L}$ of a fluorochrome solution prepared by mixing $1.5 \mu \mathrm{L}$ of Syto9 stain and $1.5 \mu \mathrm{L}$ of propidium iodide (PI) with $1 \mathrm{~mL}$ of ultrapure water. Samples were then incubated in dark for $10 \mathrm{~min}$ prior to microscopic analysis. Surfaces were kept in a humid environment during the experiments to prevent drying. Samples were examined with an epifluorescence microscope (AXIO 100 Zeiss), images were acquired with a 10x or 40x objective lens and a CCD camera (AxioCamMRm Zeiss). Fluorochromes were respectively excited and detected at $455-495 \mathrm{~nm}$ and $505-555 \mathrm{~nm}$ for Syto9 and at 533-558 nm and 570-640 nm for PI. To obtain statistically relevant data; about 10 different locations of each surface were analyzed, at least a thousand bacteria were enumerated and experiments were repeated on three independent sets of samples. Bacterial counting (red, damaged membrane; green, intact membrane) was done using the software ImageJ and the viability calculated as follows: Viability $(\%)=100 \times($ number of green bacteria $) /($ number of green bacteria + number of red bacteria).

\section{Evaluation of bacteria damaging by scanning electronic microscopy}

After deposition and incubation for 3 hours on the different surfaces, bacteria were fixed using formaldehyde at $37 \%$ to avoid collapsing of cells upon drying. After 15 min, samples were washed six times with ultrapure water to remove non-adhered bacteria, dehydrated in successive baths of increasing ethanoic concentration $(25,50,75,100 \%)$ and dried under a laminar air flow. SEM images were recorded with a Hitachi SU-70 field emission gun scanning electron microscope. The samples were fixed on an alumina SEM support with a carbon adhesive tape and were observed without metallization. An in-lens secondary electron detector (SEUpper) was used to characterize our samples. The accelerating voltage was $1 \mathrm{kV}$, and the working distance was around $5 \mathrm{~mm}$. At least five different locations were analyzed on each surface, arising to the observation of a minimum of 100 single bacteria observed.

\section{Cultivability (bacterial growth capacity)}

In order to evaluate the bacterial growth capacity after being in contact with the glycosylated substrates, each inoculated sample was transferred into a sterile tube containing $2 \mathrm{~mL}$ of isotonic sterile solution $(\mathrm{NaCl}$ at $0.9 \%)$ and sonicated $5 \mathrm{~min}$. The obtained bacterial 
suspensions were diluted 10, 100 and 1000 times and a volume of $50 \mu \mathrm{L}$ of each was deposited in duplicate on Petri dishes filled with BHI $+\operatorname{agar}(37 \mathrm{~g} / \mathrm{L}+15 \mathrm{~g} / \mathrm{L}$, respectively). These plates were incubated at $37^{\circ} \mathrm{C}$ overnight before enumeration. Results are expressed in percentages of the number of attached and cultivable bacterial cells onto the glycosylated surfaces as compared to control gold substrates: Cultivability $(\%)=100 \times($ number of colonies forming unity on sample)/(number of colonies forming unity on control gold substrate). These tests were done in triplicate and the cultivability value was averaged over the three samples. The uncertainty attached to these results follows from the statistical analysis of these repeated experiments.

\section{RESULTS}

\section{Glycolipids grafting on Au (111)}

Glycosylation of gold surfaces was performed using the glycolipids shown in Figure 1. This work explores the molecular variations of the oleic acid derivative of sophorolipids (glucose $\beta 1,2)^{26}$ in terms of both the carbohydrate moiety and fatty acid: glucolipid (GL) and acetylated sophorolipid ( $\mathrm{SL}_{\mathrm{a}}$ ) consist of an oleic acid linked respectively to a single glucose unit and an acetylated sophorose headgroup ${ }^{39}$; branched sophorolipid $\left(\mathrm{SL}_{\mathrm{b}}\right)$ carries a deacetylated acidic sophorolipid linked by a glycosidic bond to the C13 position of 13hydroxydocosanoic acid. Elaidic derivatives of trans configuration sophorolipids $\left(\mathrm{SL}_{\mathrm{t}}\right)$ have also been used. The stearic acid derivatives of sophorolipids (referred as $\mathrm{SL}_{0}$ ), as well as the more classical oleic acid sophorolipids (SL), studied in our previous works, are also presented for comparison. ${ }^{26}$ Finally, cellobioselipid (CL) and its hydrolyzed variant $\left(\mathrm{CL}_{h}\right)$ have also been used. ${ }^{41}$ These glycolipids are composed of a fully saturated C16 hydroxylated fatty acid and a cellobiose moiety (glucose $\beta 1,4)$.

All glycolipids have been individually grafted, via their carboxylic acid, onto a selfassembled monolayer (SAM) of cysteamine (cys) formed on a flat gold substrate, according to a previously described protocol. ${ }^{25,26}$ The glycolipid-functionalized surfaces were characterized by infrared spectroscopy (PM-RAIRS) and X-ray photoelectron spectroscopy (XPS). The corresponding spectroscopic data are given in Figure S1 and Figure S2 where the attribution is given in corresponding captions, on the basis of ref. ${ }^{25,26}$. Both techniques demonstrate the anchoring of glycolipids by amide bonds ${ }^{47}$. The presence of aliphatic chains and carbohydrates is also attested by both vibrational bands ${ }^{48}$ and XPS spectra. ${ }^{49-52}$ The elongation of the $\mathrm{C}=\mathrm{O}$ bonds at $1740 \mathrm{~cm}^{-1}$ on acetyl moieties produces an additional signal on the infrared spectra of substrates prepared with acetylated $\mathrm{SL}_{\mathrm{a}}$ and CL. 
While both PM-RAIRS and XPS demonstrate the success of glycolipids grafting, XPS analyses can also be used to estimate the thickness of the cysteamine and glycolipid coatings, which are assumed to form a homogeneous layer on the gold. This structural parameter can be obtained by considering the signal of a component of the layer (e.g. $\mathrm{C}=\mathrm{O}, \mathrm{N}$ or $\mathrm{S}_{\text {bound }}$ signal for instance) and that of the substrate (Au), using the methodology outlined in ref. ${ }^{25}$. The thickness estimate for each glycolipid is summarized in Figure 2A.The thickness of the cys on gold is about $3 \pm 1 \AA$, which corresponds to the formation of a short chain $\mathrm{SAM}^{48}$, while fatty acid (oleic, stearic, elaidic) controls, grafted onto the cysteamine, provide a layer of about $17 \AA$, as expected for $\mathrm{C} 18$ molecules ${ }^{25,26}$. Layer thicknesses of grafted glycolipids appear around $30 \AA$ on average, which is consistent with the thickness measured by ellipsometry on monolayers of saccharide-functionalized alkanethiols. ${ }^{50}$ Sophoro- and cellobioselipids layers containing disaccharides are thicker than $28 \AA$, whereas GL, which contains only a single glucose headgroup, forms a thinner layer of $22 \pm 3 \AA$. Furthermore, the difference of about $6 \AA$ observed in the thicknesses between a layer of GL $(22 \pm 3 \AA)$ and SL $(28 \pm 2 \AA)$ is intermediate between the length of a single glucose unit (about $10 \AA^{53,54}$ ) and the ellipsometric difference of $3 \AA$ between monosaccharides and disaccharides adsorbed monolayers. ${ }^{50}$ Differences among all glycolipids layers containing disaccharides (from $28 \pm 2 \AA$ for SL up to $37 \pm 4 \AA$ for CL) can be attributed to the variations in the carbohydrate headgroups, which may also contribute to changes in the configuration and density of the monolayers, although these are not obvious to determine here. ${ }^{48,55,56}$ A difference of a few Ångströms systematically characterizes the $\mathrm{SL}_{\mathrm{a}}$ (33 $\pm 3 \AA$ ) and CL ( $37 \pm 4 \AA$ ) layers, both containing either acetyl groups or short organic acid chain on the disaccharide, compared to the corresponding hydrolyzed compounds, SL ( $28 \pm 2$ $\AA)$ and $\mathrm{CL}_{\mathrm{h}}(32 \pm 2 \AA)$ respectively. The wettability of all glycosylated surfaces was also examined and the water contact angle (WCA) was measured on each substrate (Figure 2B). Surfaces bearing only a fatty acid, or monosaccharide (GL), appear slightly less hydrophilic (with WCA between $65 \pm 2^{\circ}$ and $68 \pm 2^{\circ}$ ) than substrates functionalized with disaccharides $\left(\mathrm{WCA} \leq 61 \pm 2^{\circ}\right.$ ), regardless of their acetylation, their linkage to the unsaturated aliphatic chain or the isometry of the unsaturated aliphatic chain. On the contrary, the surfaces of saturated glycolipids $\left(\mathrm{SL}_{0}, \mathrm{CL}, \mathrm{CL}_{\mathrm{h}}\right)$ have larger WCAs $\left(\geq 70 \pm 2^{\circ}\right)$, as already observed before, and this may indicate a variation in the orientation of the sugars. ${ }^{26}$ 

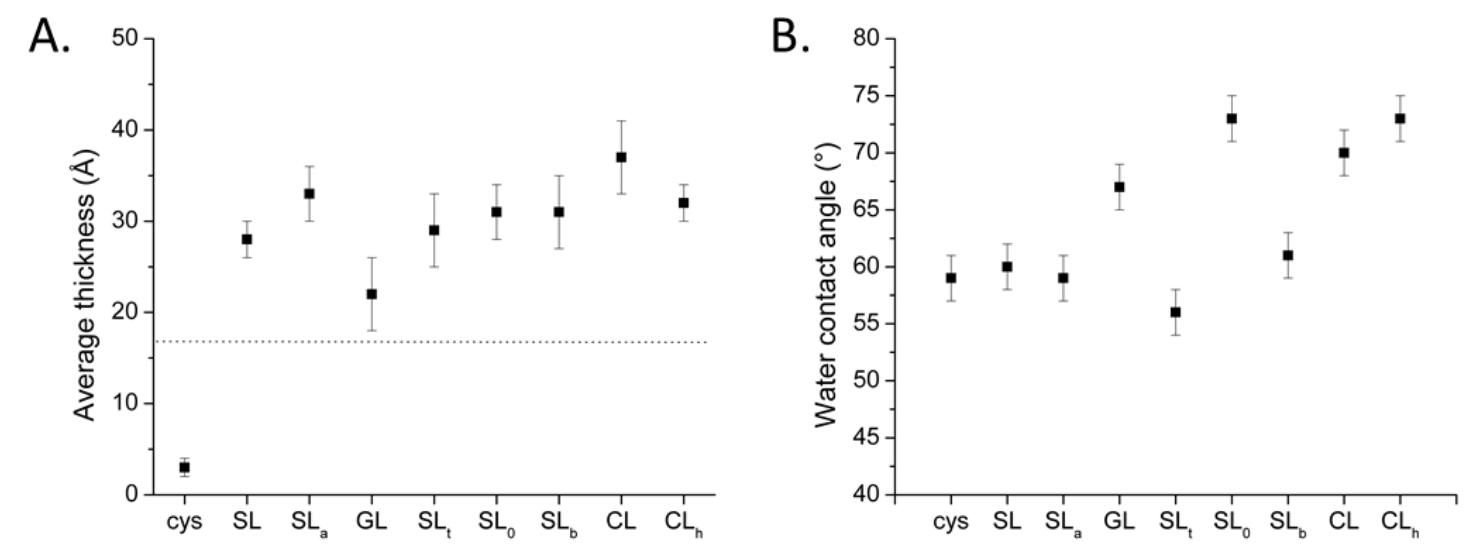

Figure 2. Physico-chemical properties of glycosylated surfaces.

(A) Thickness and (B) water contact angle measured on self-assembled monolayers of cysteamine (cys), deacetylated sophorolipid (SL), cis C18 sophorolipid (SL ( $_{\mathrm{a}}$, glucolipid (GL), trans C18 sophorolipid $\left(\mathrm{SL}_{\mathrm{t}}\right)$, branched sophorolipid $\left(\mathrm{SL}_{\mathrm{b}}\right)$, cellobioselipid $(\mathrm{CL})$, the saturated stearic acid sophorolipid $\left(\mathrm{SL}_{0}\right)$ and hydrolyzed cellobioselipid $\left(\mathrm{CL}_{\mathrm{h}}\right)$. The dotted line indicates the average thickness of corresponding sugar-free lipids (oleic acid, stearic acid, elaidic acid) monolayers.

\section{Antimicrobial activity}

The biocidal effect of grafted oleic acid sophorolipids (SL) was tested against various Gram-positive and Gram-negative bacteria and it was shown that in the best case, depending on the type of microorganism, between $40 \%$ and $50 \%$ of the bacterial population was affected by the SL coating. ${ }^{25,26}$ Gram-positive bacteria appeared more susceptible and the nonpathogenic (to human) Listeria ivanovii strain was selected in this work as a standard. The protocol we developed to assess the biocidal activity of coated substrates involved the evaluation of bacterial adhesion to glycosylated surfaces and the resulting bacterial cultivability. Cultivability below adhesion indicates a biocidal effect against bacteria. These results were compared to the integrity of the bacterial membrane read using the Live/Dead fluorescent staining kit. All these data are normalized by the references obtained on biocompatible gold surfaces and are shown in Figure 3. The primer-modified gold substrate (cys) is a glycolipid-free control. 


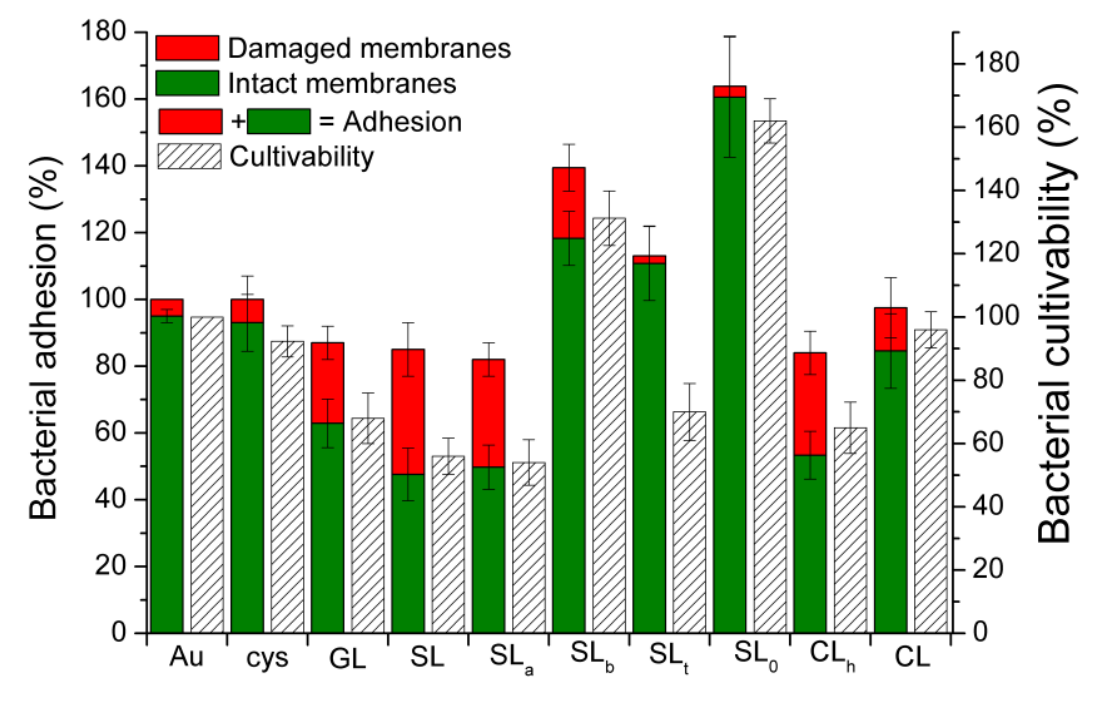

Figure 3. Antimicrobial properties of glycosylated surfaces.

Adhesion (bicolor bars, left axis) and cultivability (dashed bars, right axis) of Listeria ivanovii bacteria deposited on self-assembled monolayers of deacetylated sophorolipid (SL), cis C18 sophorolipid ( $\mathrm{SL}_{\mathrm{a}}$ ), glucolipid (GL), trans $\mathrm{C} 18$ sophorolipid $\left(\mathrm{SL}_{\mathrm{t}}\right)$, branched sophorolipid $\left(\mathrm{SL}_{\mathrm{b}}\right)$, cellobioselipid $(\mathrm{CL})$, the saturated stearic acid sophorolipid $\left(\mathrm{SL}_{0}\right)$ and hydrolyzed cellobioselipid $\left(\mathrm{CL}_{\mathrm{h}}\right)$.

The results are relative to the same experiments performed on a bare gold substrate. The adhesive bars are colored according to the ratio of damaged (red) and intact (green) bacteria determined using the Live/Dead@ kit.

Biocompatile gold $(\mathrm{Au})$ surfaces and cysteamine (cys) monolayers constitute negative controls.

\section{Biocidal properties of glycosylated surfaces}

Bacterial adhesion is on average $15 \%$ lower on the glycosylated surfaces than on the negative control (Figure 3, red/green bars, left scale), confirming, for longer time scales (>1 $\mathrm{h}$ ), the antiadhesive effects attested at the nanoscale by single cell force microscopy. ${ }^{24}$ Bacterial cultivability after contact with the substrates (Figure 3, dashed bars, right scale) matches bacterial adhesion for negative controls (Au, cys) and for $\mathrm{SL}_{0}$ and CL samples, indicating that no biocidal effect can be observed for the latter compounds. On the contrary, cultivability is systematically lower than adhesion for GL, SL, SLa, $\mathrm{SL}_{t}$ and $\mathrm{CL}_{\mathrm{h}}$, suggesting a biocidal effect for these compounds. Furthermore, the decreases in cultivability are consistent with the fraction of the bacterial population with membrane damages (red portion of bicolor bars in Figure 3, left scale; an overview of images obtained using the Live/Dead staining kit is given in Figure S3), 
suggesting that the biocidal effect of glycosylated surfaces is always due to membrane perturbations and alterations, which could be observed qualitatively by electron microscopy (Figure S4). It should be noted that grafted-glucose can damage some bacteria although they are capable of fermenting glucose units when they are free in solution ${ }^{57}$. $\mathrm{CL}_{h}$ surfaces show a similar biocidal effect (35\% reduction in bacterial cultivability correlated to $32 \%$ of membranes-damaged cells). Thus, the antimicrobial properties of glycolipids surfaces are not directly correlated to sophorose itself, nor to the amount of glucose units, and they are not affected by the carbohydrate linkage in the glucidic moiety (sophorose being characterized by a $\beta 1,2$ glucose link, whereas $C_{L}$ has a deacetylated cellobiose ( $\beta 1,4$ glucose) headgroup).

\section{Effect of the carbohydrate valency on the biocidal properties}

The number of glucose units appears as a key factor and grafted monosaccharides appear to be less active than disaccharides: the proportion of damaged bacteria is reduced by one third on GL coated substrates compared to the surfaces functionalized with the disaccharide-containing SL, of which the biocidal efficiency has previously been shown to be dependent to the surface density ${ }^{25}$. These results are in line with various studies highlighting the fact that the sugar density, as well as the sugar valency, are key parameters of carbohydrate properties and drive their interactions with the biomolecules ${ }^{58,59}$ and biomembranes ${ }^{60}$. At low concentration, sugars may bind quite strongly to lipid bilayers and perturb their organization by their accumulation at the interface, while at high concentration sugars gradually become expelled from the membrane surface, and this repulsive mode of interaction counteracts membrane disturbance ${ }^{61}$. Besides, the architecture of multivalent ligands is known to influence their affinities to receptors and to lead them to act either as effective inhibitors of protein binding, or as effectors of receptors clustering. For instance, high molecular weight polydisperse polyvalent mannose-bearing ligands inhibit the protein concanavalin A (Con A) binding, while linear oligomeric ligands favor clustering of this receptor. ${ }^{58}$ Studies on glycopolymers and glycodendrimers also demonstrate that not only the number of ligand groups matters, but also the spacer connecting the sugar to the scaffold. ${ }^{61}$ The effect of the carbohydrate valency observed here is also reminiscent to the charge-density threshold for optimal efficiency of biocide cationic surfaces. ${ }^{62}$

\section{Effect of the glycolipid conformation on the biocidal properties}

Data in Figure 3 also provide some pieces of information on the conformational effects of carbohydrates after grafting on the surface. While SL, $\mathrm{SL}_{0}$ and $\mathrm{SL}_{t}$ coatings display the same 
headgroup (deacetylated sophorose) and similar layer thickness strong differences are observed in their biocidal properties. These latter are then attributed to conformation changes expected among monounsaturated cis and trans isomers, and saturated chains. One can reasonably consider that the saturation/unsaturation features of the lipid backbone play an important role. The conformation of the SL aliphatic chain is known to be important: our previous study, confirmed here, demonstrates that saturated $\mathrm{SL}_{0}$ coatings do not exhibit any antimicrobial properties ${ }^{26}$ contrary to the monounsaturated SL coatings. The trans isomer $\mathrm{SL}_{\mathrm{t}}$ exhibits intermediate properties between the cis isomer of SL and the saturated molecules: it seems to promote the microbial adhesion (increased up to 113\%) but it is responsible for a decrease of their cultivability (of $30 \%$ ).

\section{Impact of the carbohydrate accessibility on the biocidal properties}

Acetylation first does not seem to play an important role, since SL and $\mathrm{SL}_{\mathrm{a}}$ have comparable biocidal effects, suggesting that the moieties on C6' and C6" positions on sophorose do not have a critical role in biocidal activity. On the contrary, the acetyl moieties and fatty acid esters carried by cellobiose units in CL sample appear to interfere the biocidal activities of the anchored molecules when compared to hydrolyzed $\mathrm{CL}_{\mathrm{h}}$.

However, it should be kept in mind that $\mathrm{SL}_{\mathrm{a}}$ carry only acetyl groups, while C6 fatty esters are covalently attached to CL, and may limit the carbohydrate accessibility. The difference in biocidal behavior between $\mathrm{CL}$ and $\mathrm{SL}_{\mathrm{a}}$ could also result from the position of the acetyl groups and the linkage of the carbohydrate units to the aliphatic chain. Unfortunately, the microbial synthesis of CL and SL derivatives does not provide all the molecular intermediates necessary for a systematic study, and synthetic compounds would be needed to discriminate the influence of the mentioned parameters. Nevertheless, the accessibility of carbohydrates moieties is certainly a key parameter when examining antibacterial activity. In the case of SLb the relative position of the sophorose units seems identical to that of SL, but the presence of the saturated lateral chain, allowing the rotation of the sophorose unit, reduces the killing efficiency to only $16 \%$ compare to $44 \%$ for $\mathrm{SL}$. However, if $\mathrm{SL}_{\mathrm{b}}$ is compared to $\mathrm{SL}_{t}$ or $\mathrm{SL}_{0}$, its activity is 5 time higher than that of the latter ones, probably due to a ratio of positioned as in SL layers.

\section{DISCUSSION}

It has been demonstrated that slight variations in the glycolipids structures lead to different behavior in solution, e.g. variations of alkyl chains or sugar acetylation of rhamnolipids affect not only their physical properties ( $\mathrm{cmc}$, surface tension, acyl chain fluidity, 
structure of the aggregated state), but also the interactions of these compounds with living organisms (ability to insert into membranes) ${ }^{63}$. Similarly, the present work shows that glycosylated substrates exhibit different antimicrobial properties depending on the carbohydrates and their anchoring to the surface. The molecular variations studied here allow the identification of certain key parameters.

The "cleavage" of sophorose into a simple glucose moiety (GL compound) decreases the killing efficiency of functionalized surfaces without affecting the mechanism of action (lysis of bacterial membranes and decrease in the cultivability of microorganisms). Thus, the number of sugars seems to be correlated with the interactions between bacteria and glycosylated surfaces. This is not surprising: the multivalence of glycoconjugate compounds (and their assemblages) is considered to be very important in developing effective interactions with other molecules: ${ }^{64}$ the higher the sugar valency, the more effective the conjugate interactions with their molecular environment, sugars and proteins in particular (the individual interactions between these compounds being relatively weak $)^{65}$. Ligand-receptor recognition phenomena, in the manner of a molecular "Velcro"TM 66 are then favored. A parallelism could be established between these works and the relative improvement in the membrane damage phenomena observed here when switching from a mono to a disaccharide. However, multivalency is probably not the only parameter to be taken into account, as the conformation of carbohydrates on the surface, the other molecular variations studied in this work, seems to be of the utmost importance.

The biocidal effect is minimized, or even disappears, when the geometry of the lipid chain is modified. Variation of the degree of saturation of the aliphatic chain (SL vs. SL $)$ affects biocidal activity, surface wettability and appears to influence the orientation of the sophorose groups. Thus, the conformation of carbohydrate units emerges as a key parameter in the interactions between sophorolipids and microorganisms. This observation suggests that the phenomena observed are not specific to sophorose per se but rather induced by the molecular arrangement and orientation of certain hydroxyl groups. Similar observations have been reported recently in the field of glycobiology. The creation of glycosylated surfaces with azobenzene-containing ligands, whose orientation can be controlled by applying a light stimulus, has demonstrated the influence of sugar orientation on bacterial adhesion. Modification of the chirality of glycosylated dendrimers has also been shown to have an impact on the adhesion of Escherichia coli. ${ }^{67}$ In these studies, variations in bacterial adhesion are interpreted as an alteration of specific recognition phenomena between dendrimers sugars and bacterial cell wall receptors. Comparison between the phenomena described in these studies 
and this work could constitute an argument for a biochemical explanation of the biocidal action of glycolipids. However, the nature of the phenomena studied here is not strictly the same. In this work, bacterial adhesion is minimal on surfaces functionalized with monounsaturated fatty acids (OA derivates), whereas samples functionalized with saturated fatty acid (SA derivates) seem rather favorable to microbial adhesion (Figure 3). Thus, the behavior of bacteria seems to be affected by the saturation of the lipid chain, even in the absence of sugars, which is not the case in other studies. ${ }^{67}$ The differences observed between surfaces functionalized with saturated and monounsaturated glycolipids may therefore not only be related to biochemical criteria. More generally, sugars are known to play a key role in the (de)stabilization of the biological system both in vivo and in vitro, either by influence the solvating shell of biomolecules or by specifically interacting with biological molecules (direct sugar-molecule binding at the interface $)^{68}$ and several studies on the antimicrobial activity of glycolipids in solution suggest biophysical, rather than biochemical, effects, as it has been discussed for cellobioselipids ${ }^{69}$. However, grafting of these compounds onto a surface limits the antimicrobial activity unless cellobioselipids are hydrolyzed: our data show that $\mathrm{CL}_{h}$ has an impact on the membrane integrity of L. ivanovii. Interestingly, the hydroxylated form of CL is considered less bioactive against fungus and bacteria in solution. ${ }^{35,70}$ These experiments confirm that the phenomena observed are not specific to sophorolipid, as previously assumed, ${ }^{26}$ but they can be reproduced on surfaces coated with other glycoconjugate molecules. The conformation of the sugars is an important parameter to consider. Specifically, the orientation and availability of specific $\mathrm{C}-\mathrm{OH}$ groups appears to be critical. Indeed, acetylation of the $\mathrm{C}-\mathrm{OH}$ groups in the 6' and 6" positions does not affect the antimicrobial activity of grafted sophorolipids, suggesting that the C6' and C6" carbons of sophorose do not play a determining role in biocidal activity, while esterification of the $2 "$ and 6' positions in cellobioselipid inactivates it.

\section{CONCLUSION}

In this work, we have shown that self-assembled monolayers of microbial-derived glycolipids can be chemically grafted onto a gold substrate using a cysteamine primer; the surfaces thus modified have antibacterial properties against $\mathrm{Gram}^{+}$bacteria Listeria ivanovii, with an efficiency that depends on the chemical and structural composition of the SL derivative. Surfaces analyses estimate thicknesses to be very close for each SL derivate, ensuring an almost identical molecular surface density for all the different substrates. The bacterial killing efficiency (corrected by the bacterial adhesion) can be up to $44 \%$ at best and as low as $2 \%$ at worst, which is close to the statistical bacterial death observed in standard controls (Au and 
cysteamine primer). From a mechanistic point of view, our results show that several parameters influence the killing efficiency and bacterial adhesion, such as the saturation and conformation of the fatty acid backbone, the number of glucose units in the head group or the sugar lateral chains. Thus, our results highlight that for sophorolipds with the same sugar head groups, the unsaturation of the fatty acid backbone is required and the cis conformation (SL) will have a higher antibacterial activity when compared to the trans conformation ( $\mathrm{SL}_{t}$; in addition, the number of glucose unit is also a key factor, a decrease of one third of the antibacterial activity is observed by omitting one glucose unit (GL) in relation to two (SL).

\section{ACKNOWLEDGMENTS}

This work was supported by French state funds managed by the ANR within the Investissements d'Avenir program under Reference ANR-11-IDEX-0004-02 and more specifically within the framework of the Cluster of Excellence MATISSE. The authors acknowledge IMPC (Institut des Matériaux de Paris Centre, FR CNRS 2482) and the C'Nano projects of the Region Ile-de-France, Omicron XPS apparatus funding. The authors would also like to thank Dr P. L. V. Baghavatula from CSIR is acknowledged for providing the $\mathrm{SL}_{\mathrm{t}}$ compound, Christophe Calers and Dr. Christophe Méthivier from LRS for their help with XPS analyses and Sandra Casale for her help in acquiring SEM-FEG data. 


\section{REFERENCES}

1. L. H. G. Morton and S. B. Surman, Int. Biodeterior. Biodegradation, 1994, 34, 203-221.

2. J. D. Bryers, Biotechnol. Bioeng., 2008, 100, 1-18.

3. N. R. B. Boase, M. D. T. Torres, N. L. Fletcher, C. d. I. Fuente-Nunez and K. E. Fairfull-Smith, Polym. Chem., 2018, 9, 5308-5318.

4. B. Meyer, Int. Biodeterior. Biodegradation, 2003, 51, 249-253.

5. A. D. Verderosa, M. Totsika and K. E. Fairfull-Smith, Front. Chem., 2019, 7, 824.

6. J. Hasan, R. J. Crawford and E. P. Ivanova, Trends Biotechnol., 2013, 31, 295-304.

7. D. M. Yebra, S. Kiil and K. Dam-Johansen, Prog. Org. Coat., 2004, 50, 75-104.

8. M. E. Forman, M. C. Jennings, W. M. Wuest and K. P. Minbiole, Chem. Med. Chem., 2016, 11, 1401-1405.

9. I. M. Banat, A. Franzetti, I. Gandolfi, G. Bestetti, M. G. Martinotti, L. Fracchia, T. J. Smyth and R. Marchant, Appl. Microbiol. Biotechnol., 2010, 87, 427-444.

10. M. Sun, J. Dong, Y. Xia and R. Shu, Microb. Pathog., 2017, 107, 212-218.

11. M. Sun, Z. Zhou, J. Dong, J. Zhang, Y. Xia and R. Shu, Microb. Pathog., 2016, 99, 196-203.

12. B. K. Yoon, J. A. Jackman, E. R. Valle-González and N.-J. Cho, Int. J. Mol. Sci., 2018, 19, 1114.

13. D. Kitamoto, H. Yanagishita, T. Shinbo, T. Nakane, C. Kamisawa and T. Nakahara, J. Biotechnol., 1993, 29, 91-96.

14. S. Lang, E. Katsiwela and F. Wagner, Fett. Wiss.Technol. Sci. Technol., 1989, 91, 363-366.

15. A. L. S. Coelho, P. E. Feuser, B. A. M. Carciofi, C. J. de Andrade and D. de Oliveira, Appl. Microbiol. Biotechnol., 2020, 104, 2297-2318.

16. L. Rodrigues, I. M. Banat, J. Teixeira and R. Oliveira, J. Antimicrob. Chemother., 2006, 104, $2297-$ 2318.

17. J. M. Farias, T. C. M. Stamford, A. H. M. Resende, J. S. Aguiar, R. D. Rufino, J. M. Luna and L. A. Sarubbo, Int. J. Biol. Macromol., 2019, 129, 853-860.

18. J. Hage-Hülsmann, A. Grünberger, S. Thies, B. Santiago-Schübel, A. S. Klein, J. Pietruszka, D. Binder, F. Hilgers, A. Domröse, T. Drepper, D. Kohlheyer, K.-E. Jaeger and A. Loeschcke, PLoS One 2018, 13, e200940.

19. C. A. Marangon, V. C. A. Martins, M. H. Ling, C. C. Melo, A. M. G. Plepis, R. L. Meyer and M. Nitschke, ACS Appl. Mater. Interfaces, 2020, 12, 5488-54989.

20. C. Ceresa, L. Fracchia, M. Williams, I. M. Banat and M. A. Díaz De Rienzo, J. Biotechnol., 2020, 309, 34-43.

21. M. Ohadi, H. Forootanfar, G. Dehghannoudeh, T. Eslaminejad, A. Ameri, M. Shakibaie and M. Adeli-Sardou, Microb. Pathog., 2020, 138, 103806.

22. Q. Shu, T. Wei, H. Lu, Y. Niu and Q. Chen, Appl. Microbiol. Biotechnol., 2020, 104, 5053-5064.

23. M. Elshikh, I. Moya-Ramírez, H. Moens, S. Roelants, W. Soetaert, R. Marchant and I. M. Banat, J. Appl. Microbiol., 2017, 123, 1111-1123.

24. C. Valotteau, N. Baccile, V. Humblot, S. Roelants, W. Soetaert, C. V. Stevens and Y. F. Dufrêne, Nanoscale Horiz., 2019, 4, 975-982.

25. C. Valotteau, I. Banat, C. Mitchell, H. Lydon, F. Babonneau, C.-M. Pradier, N. Baccile and V. Humblot, Coll. Surf. B, 2017, 157, 325-334.

26. C. Valotteau, C. Calers, S. Casale, J. Berton, C. V. Stevens, F. Babonneau, C.-M. Pradier, V. Humblot and N. Baccile, ACS Appl. Mater. Interfaces, 2015, 7, 18086-18095.

27. V. Shah, D. Badia and P. Ratsep, Antimicrob. Agents Chemother., 2007, 51, 397-400.

28. N. Laurent, J. VogImeir and S. L. Flitsch, Chem. Comm., 2008, 37, 4400-4412.

29. K. Larsen, M. B. Thygesen, F. Guillaumie, W. G. T. Willats and K. J. Jensen, Carbohydr. Res., 2006, 341, 1209-1234.

30. R. Liang, J. Loebach, N. Horan, M. Ge, C. Thompson, L. Yan and D. Kahne, Proc. Natl. Acad. Sci. USA, 1997, 94, 10554-10559. 
31. R. T. Otto, H. J. Daniel, G. Pekin, K. Müller-Decker, G. Fürstenberger, M. Reuss and C. Syldatk, Appl. Microbiol. Biotechnol., 1999, 52, 495-501.

32. M. R. d. Oliveira, A. Magri, C. Baldo, D. Camilios-Neto, T. Minucelli and M. A. P. C. Celligoi, Int. J. Adv. Biotechnol. Res., 2015, 6, 161-174.

33. U. Rau, R. Heckmann, V. Wray and S. Lang, Biotechnol. Lett., 1999, 21, 973-977.

34. K. M. Saerens, L. Saey and W. Soetaert, Biotechnol. Bioeng., 2011, 108, 2923-2931.

35. E. Kulakovskaya, B. Baskunov and A. Zvonarev, J. Oleo Sci., 2014, 63, 701-707.

36. N. Baccile, A.-S. Cuvier, C. Valotteau and I. N. A. Van Bogaert, Eur. J. Lipid. Sci. Technol., 2013, 115, 1404-1412.

37. A.-S. Cuvier, J. Berton, C. V. Stevens, G. C. Fadda, F. Babonneau, I. N. Van Bogaert, W. Soetaert, G. Pehau-Arnaudet and N. Baccile, Soft Matter, 2014, 10, 3950-3959.

38. K. Ciesielska, I. N. V. Bogaert, S. Chevineau, B. Li, S. Groeneboer, W. Soetaert, Y. V. d. Peer and B. Devreese, J. Proteomics, 2014, 98, 159-174.

39. N. Baccile, F. Babonneau, I. M. Banat, K. Ciesielska, A.-S. Cuvier, B. Devreese, B. Everaert, H. Lydon, R. Marchant, C. A. Mitchell, S. Roelants, L. Six, E. Theeuwes, G. Tsatsos, G. E. Tsotsou, B. Vanlerberghe, I. N. A. V. Bogaert and W. Soetaert, ACS Sustain. Chem. Eng., 2017, 5, 11861198.

40. K. M. J. Saerens, J. Zhang, L. Saey, I. N. A. V. Bogaert and W. Soetaert, Yeast, 2011, 28, 279-292.

41. N. Baccile, M. Selmane, P. L. Griel, S. Prévost, J. Perez, C. V. Stevens, E. Delbeke, S. Zibek, M. Guenther, W. Soetaert, I. N. A. V. Bogaert and S. Roelants, Langmuir, 2016, 32, 6343-6359.

42. P. Dhasaiyan, S. Prévost, N. Baccile and B. L. V. Prasad, Langmuir, 2018, 34, 2121-2131.

43. N. Baccile, P. L. Griel, S. Prévost, B. Everaert, I. N. A. V. Bogaert, S. Roelants and W. Soetaert, ChemistryOpen, 2017, 6, 526-533.

44. A. P. Tulloch, A. Hill and J. F. T. Spencer, Can. J. Chem., 1968, 46, 3337-3351.

45. S. Spoeckner, V. Wray, M. Nimtz and S. Lang, Appl. Microbiol. Biotech., 1999, 51, 33-39.

46. J. H. Scofield, J. Electron. Spectrosc. Relat. Phenom., 1976, 8, 129-137.

47. J. Yala, P. Thebault, A. Hequet, V. Humblot, C.-M. Pradier and J. Berjeaud, Appl. Microbiol. Biotechnol., 2011, 89, 623-634.

48. M. D. Porter, T. B. Bright, D. L. Allara and C. E. D. Chidsey, J. Am. Chem. Soc., 1987, 109, 35593568.

49. R. G. Nuzzo, L. H. Dubois and D. L. Allara, J. Am. Chem. Soc., 1990, 112, 558-569.

50. T. Fyrner, H.-H. Lee, A. Mangone, T. Ekblad, M. E. Pettitt, M. E. Callow, J. A. Callow, S. L. Conlan, R. Mutton, A. S. Clare, P. Konradsson, B. Liedberg and T. Ederth, Langmuir, 2011, 27, 1503415047.

51. M. Hederos, P. Konradsson and B. Liedberg, Langmuir, 2005, 21, 2971-1980.

52. J. S. Stevens and S. L. M. Schroeder, Surf. Inter. Anal., 2009, 41, 453-462.

53. S. Abel, F.-Y. Dupradeau, E. P. Raman, A. D. MacKerell, Jr. and M. Marchi, J. Phys. Chem. B, 2011, 115, 487-499.

54. L. R. Winther, J. Qvist and B. Halle, J. Phys. Chem. B, 2012, 116, 9196-9207.

55. F. Tielens, D. Costa, V. Humblot and C. M. Pradier, J. Phys. Chem. C, 2008, 112, 182-190.

56. F. Tielens, V. Humblot, C. M. Pradier, M. Calatayud and F. Illas, Langmuir, 2009, 25, 9980-9985.

57. https://www.vetbact.org/index.php?artid=168\&vbsearchstring=Listeria\%20ivanovii.

58. C. W. Cairo, J. E. Gestwicki, M. Kanai and L. L. Kiessling, J. Am. Chem. Soc., 2002, 124, 16151619.

59. M. C. Galan, P. Dumy and O. Renaudet, Chem. Soc. Rev., 2013, 42, 4599-4612.

60. H. D. Andersen, C. Wang, L. Arleth, G. H. Peters and P. Westh, Proc. Natl. Acad. Sci. USA, 2011, 108, 1874-1878.

61. T. R. Branson and W. B. Turnbull, Chem. Soc. Rev., 2013, 42, 4613-4622.

62. R. Kugler, O. Bouloussa and F. Rondelez, Microbiology, 2005, 151, 1341-1348.

63. J. Howe, J. Bauer, J. Andrä, A. B. Schromm, M. Ernst, M. Rössle, U. Zähringer, J. Rademann and K. Brandenburg, FEBS J., 2006, 273, 5101-5112. 
64. A. Bernardi, J. Jiménez-Barbero, A. Casnati, C. D. Castro, T. Darbre, F. Fieschi, J. Finne, H. Funken, K.-E. Jaeger, M. Lahmann, T. K. Lindhorst, M. Marradi, P. Messner, A. Molinaro, P. V. Murphy, C. Nativi, S. Oscarson, S. Penadés, F. Peri, R. J. Pieters, O. Renaudet, J.-L. Reymond, B. Richichi, J. Rojo, F. Sansone, C. Schäffer, W. B. Turnbull, T. Velasco-Torrijos, S. Vidal, S. Vincent, T. Wennekes, H. Zuilhof and A. Imberty, Chem. Soc. Rev., 2013, 42, 4709-4727.

65. A. Martinez, C. Ortiz Mellet and J. M. Garcia Fernandez, Chem. Soc. Rev., 2013, 42, 4746-4773.

66. R. J. Pieters, Org. Biomol. Chem., 2009, 7, 2013-2025.

67. E. M. V. Johansson, R. U. Kadam, G. Rispoli, S. A. Crusz, K.-M. Bartels, S. P. Diggle, M. Camara, P. Williams, K.-E. Jaeger, T. Darbre and J.-L. Reymond, Med. Chem. Comm., 2011, 2, 418-420.

68. K. B. Konov, D. V. Leonov, N. P. Isaev, K. Y. Fedotov, V. K. Voronkova and S. A. Dzuba, J. Phys. Chem. B, 2015, 119, 10261-10266.

69. B. Mimee, R. Pelletier and R. R. Belanger, J. Appl. Microbiol., 2009, 107, 989-996.

70. T. Kulakovskaya, A. Shashkov, E. Kulakovskaya, W. Golubev, A. Zinin, Y. Tsvetkov, A. Grachev and N. Nifantiev, J. Oleo Sci., 2009, 58, 133-140. 


\section{FIGURE LEGENDS}

Figure 1. Set of microbial glycolipids presenting rational variation of the glycosidic headgroup (horizontally) and chain configuration (vertically).

The different acid chains are shown in grey as references.

3 glycolipids have a cis $\mathrm{C} 18$ oleic acid (OA) chain: the typical sophorolipid (SL), the acetylated sophorolipid $\left(\mathrm{SL}_{\mathrm{a}}\right)$ and the glucolipid (GL).

The trans sophorolipid $\left(\mathrm{SL}_{\mathrm{t}}\right)$ has a trans $\mathrm{C} 18$ elaidic acid (EA) chain.

The last glycolipids have a fully saturated chain: the saturated stearic acid (SA) sophorolipid $\left(\mathrm{SL}_{0}\right)$, the branched sophorolipid $\left(\mathrm{SL}_{\mathrm{b}}\right)$, the cellobioselipid $(\mathrm{CL})$ and the hydrolysed cellobioselipid $\left(\mathrm{CL}_{h}\right)$. For the latter, $\mathrm{R} 1=\mathrm{H}$ or $\mathrm{OH}$ and $\mathrm{R} 2=\mathrm{H}$ or $\mathrm{OH}$, as described in the materials and method section.

\section{Figure 2. Physico-chemical properties of glycosylated surfaces.}

(A) Thickness and (B) water contact angle measured on self-assembled monolayers of cysteamine (cys), deacetylated sophorolipid (SL), cis C18 sophorolipid (SLa), glucolipid (GL), trans $\mathrm{C} 18$ sophorolipid $\left(\mathrm{SL}_{\mathrm{t}}\right)$, branched sophorolipid ( $\left.\mathrm{SL}_{\mathrm{b}}\right)$, cellobioselipid $(\mathrm{CL})$, the saturated stearic acid sophorolipid $\left(\mathrm{SL}_{0}\right)$ and hydrolyzed cellobioselipid $\left(\mathrm{CL}_{\mathrm{h}}\right)$. The dotted line indicates the average thickness of corresponding sugar-free lipids (oleic acid, stearic acid, elaidic acid) monolayers ${ }^{25}$.

\section{Figure 3. Antimicrobial properties of glycosylated surfaces.}

Adhesion (bicolor bars, left axis) and cultivability (dashed bars, right axis) of Listeria ivanovii bacteria deposited on self-assembled monolayers of deacetylated sophorolipid (SL), cis C18 sophorolipid $\left(\mathrm{SL}_{\mathrm{a}}\right)$, glucolipid $(\mathrm{GL})$, trans $\mathrm{C} 18$ sophorolipid $\left(\mathrm{SL}_{\mathrm{t}}\right)$, branched sophorolipid $\left(\mathrm{SL}_{\mathrm{b}}\right)$, cellobioselipid $(\mathrm{CL})$, the saturated stearic acid sophorolipid $\left(\mathrm{SL}_{0}\right)$ and hydrolyzed cellobioselipid $\left(\mathrm{CL}_{h}\right)$.

The results are relative to the same experiments performed on a bare gold substrate. The adhesive bars are colored according to the ratio of damaged (red) and intact (green) bacteria determined using the Live/Dead@ kit.

Biocompatile gold (Au) surfaces and cysteamine (cys) monolayers constitute negative controls.

Figure S1. PM-RAIRS spectra of self-assembled monolayers of glucolipid (GL), cis C18 sophorolipid ( $\mathrm{SL}_{\mathrm{a}}$ ), branched sophorolipid $\left(\mathrm{SL}_{\mathrm{b}}\right)$, trans $\mathrm{C} 18$ sophorolipid $\left(\mathrm{SL}_{\mathrm{t}}\right)$, cellobioselipid 
(CL) and hydrolyzed cellobioselipid $\left(\mathrm{CL}_{\mathrm{h}}\right)$. The resonance signal at $1648 \mathrm{~cm}^{-1}$ ( $v_{\mathrm{C}=\mathrm{O}}$ in amide) and $1562 \mathrm{~cm}^{-1}\left(v_{\mathrm{CN}}\right.$ and $\left.\delta_{\mathrm{NH}}\right)$ are characteristic of amide bonds. The presence of aliphatic chains is attested by vibrational bands at $3000-2800 \mathrm{~cm}^{-1}\left(v_{\mathrm{S}}\right.$ and $v_{\mathrm{AS}}$ of $\mathrm{CH}_{2}$ and $v_{\mathrm{S}}$ of $\mathrm{CH}_{3}$ ) and 1400 $\mathrm{cm}^{-1}\left(\omega_{\mathrm{CH} 2}\right)$. The signal of carbohydrates resonates at $1200-1000 \mathrm{~cm}^{-1}\left(\delta_{\mathrm{CO}}\right)$, where a multitude of bands are partially overlapped and therefore difficult to interpret in detail. The elongation of the $\mathrm{C}=\mathrm{O}$ bonds on acetyl moieties produces an additional band at $1740 \mathrm{~cm}^{-1}$ on spectra of substrates prepared with acetylated $\mathrm{SL}_{\mathrm{a}}$ and $\mathrm{CL}$

Figure S2. High resolution standardized XPS spectra of the C1s (left) and N1s (right) regions recorded on self-assembled monolayers of glucolipid (GL), cis C18 sophorolipid (SLa), branched sophorolipid $\left(\mathrm{SL}_{\mathrm{b}}\right)$, trans $\mathrm{C} 18$ sophorolipid $\left(\mathrm{SL}_{\mathrm{t}}\right)$, cellobioselipid $(\mathrm{CL})$ and hydrolyzed cellobioselipid $\left(\mathrm{CL}_{\mathrm{h}}\right)$. The peak at $284.8 \pm 0.1 \mathrm{eV}$ in the $\mathrm{C} 1 \mathrm{~s}$ region attests the presence of an aliphatic chains while the two contributions, at $286.4 \pm 0.1 \mathrm{eV}(\mathrm{C}-\mathrm{OH})$ and 287.7 $\pm 0.1 \mathrm{eV}(\mathrm{O}-\mathrm{C}-\mathrm{O})$ are the signature of the carbohydrate groups, also visible in the $\mathrm{O} 1 \mathrm{~s}$. The shift of the nitrogen signal from $401.8 \pm 0.1 \mathrm{eV}\left(\mathrm{NH}_{3}{ }^{+}\right)$on cys primer layer towards $399.9 \pm 0.1 \mathrm{eV}$ $\left(\mathrm{NH}_{2}\right.$ and $\mathrm{NH}$ in amide) after immersion on glycolipids solution demonstrate that the glycolipids are grafted via amide bonds.

Figure S3. Fluorescent staining evidences membrane damages of bacteria (L. ivanovii) deposited on self-assembled monolayers of glucolipid (GL), deacetylated sophorolipid (SL), cis C18 sophorolipid ( $\left.\mathrm{SL}_{\mathrm{a}}\right)$, trans C18 sophorolipid $\left(\mathrm{SL}_{\mathrm{t}}\right)$, saturated sophorolipid $\left(\mathrm{SL}_{0}\right)$, hydrolyzed cellobioselipid $\left(\mathrm{CL}_{h}\right)$ and cellobioselipid (CL).

Biocompatile gold ( $\mathrm{Au}$ ) surfaces and cysteamine (cys) monolayers constitute negative controls. The chart above each image represents the proportion of adhering intact (percentage given) and damaged bacteria according to fluorescent staining.

Figure S4. Scanning electron microscopy reveals qualitative morphological alterations (highlighted by white arrows) of bacteria (L. ivanovii) deposited on self-assembled monolayers of glucolipid (GL), deacetylated sophorolipid (SL), cis C18 sophorolipid (SLa), trans C18 sophorolipid $\left(\mathrm{SL}_{\mathrm{t}}\right)$, saturated sophorolipid $\left(\mathrm{SL}_{0}\right)$, cellobioselipid $(\mathrm{CL})$ and hydrolyzed cellobioselipid $\left(\mathrm{CL}_{h}\right)$.Biocompatile gold $(\mathrm{Au})$ surfaces and cysteamine (cys) monolayers constitute negative controls. 


\section{Supplementary information}

\section{Antibacterial properties of glycosylated surfaces: variation of the glucosidal moiety and fatty acid conformation of grafted microbial glycolipids}

Claire Valotteau ${ }^{1,2}$, Sophie Roelants ${ }^{3,4}$, Prabhu Dasaiyan ${ }^{5}$, Susanne Zibek ${ }^{6}$, Michael Günther ${ }^{7}$, Wim Soetaert ${ }^{3,4}$, Bernd Everaert ${ }^{4}$, Claire-Marie Pradier ${ }^{1}$, Florence Babonneau ${ }^{2}$, Niki Baccile ${ }^{2, \$}$, Vincent Humblot ${ }^{1, \$, \#}$.

${ }^{1}$ Sorbonne Université, Laboratoire de Réactivité de Surface (LRS), UMR CNRS 7197, 4 place Jussieu, Paris, F-75005, France.

${ }^{2}$ Sorbonne Université, Laboratoire de Chimie de la Matière Condensée de Paris (LCMCP), UMR CNRS 7574, 4 place Jussieu, Paris, F-75005, France.

${ }^{3}$ Ghent University, Centre for Industrial Biotechnology and Biocatalysis (InBio.be), Coupure Links 653, B-9000 Gent, Belgium

${ }^{4}$ Bio Base Europe Pilot Plant, Rodenhuizekaai 1, B-9000 Gent, Belgium

${ }^{5}$ Physical and Materials Chemistry Division, CSIR - National Chemical Laboratory, Pune - 411008 , India

${ }^{6}$ Fraunhofer Institute for Interfacial Engineering and Biotechnology IGB, Nobelstraße 12, D-70569 Stuttgart, Germany

${ }^{7}$ Universität Stuttgart, Institut für Grenzflächenverfahrenstechnik, Nobelstraße 12, D-70569 Stuttgart, Germany

${ }^{\$}$ Corresponding authors: niki.baccile@ sorbonne-universite.fr; vincent.humblot@femto-st.fr \# present address: FEMTO-ST Institute, UMR CNRS 6174, Université Bourgogne Franche-Comté, 15B avenue des Montboucons, 25030 Besançon Cedex, France. 


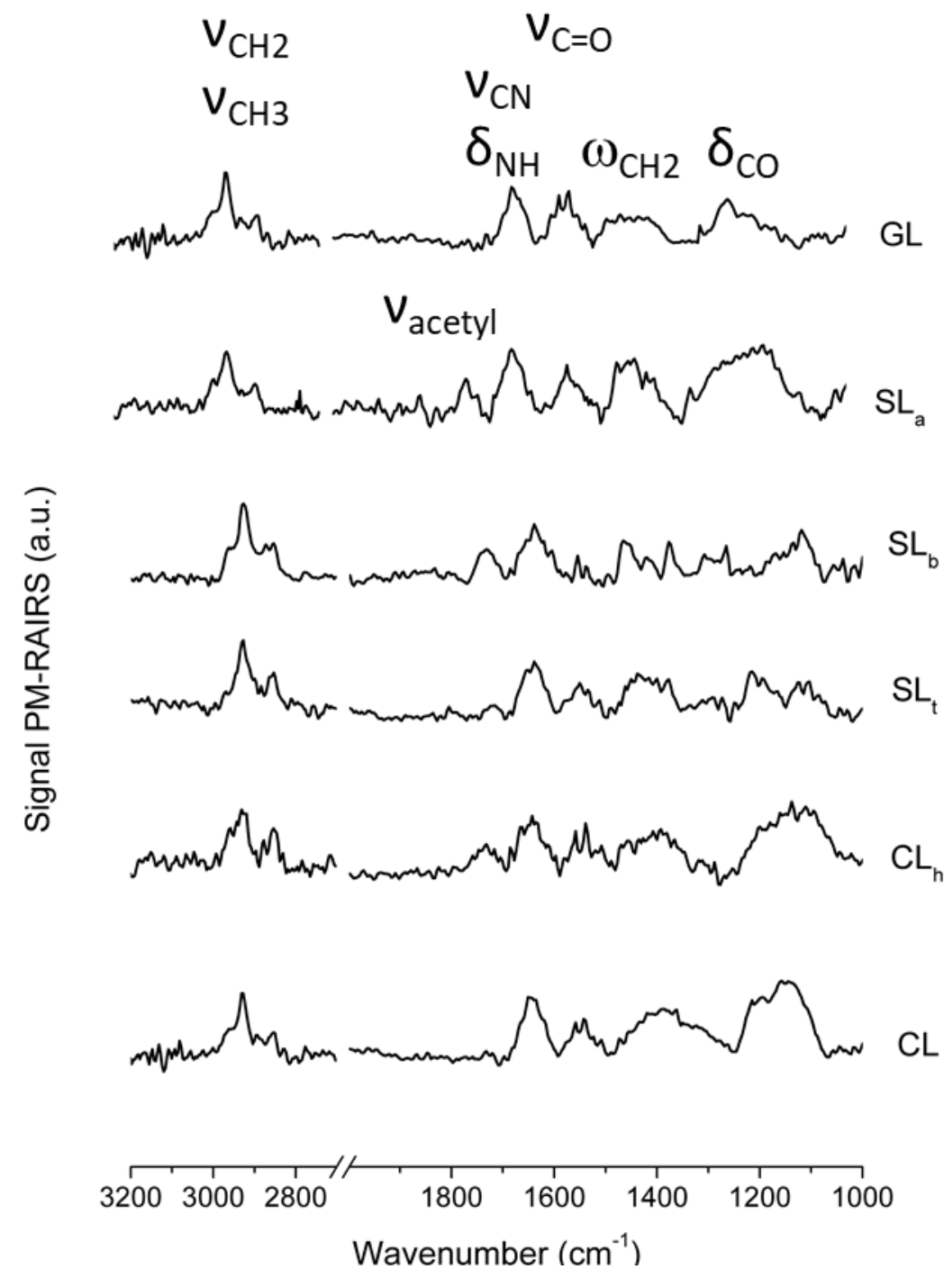

Figure S1. PM-RAIRS spectra of self-assembled monolayers of glucolipid (GL), cis C18 sophorolipid $\left(\mathrm{SL}_{\mathrm{a}}\right)$, branched sophorolipid $\left(\mathrm{SL}_{\mathrm{b}}\right)$, trans $\mathrm{C} 18$ sophorolipid $\left(\mathrm{SL}_{\mathrm{t}}\right)$, cellobioselipid $(\mathrm{CL})$ and hydrolyzed cellobioselipid $\left(\mathrm{CL}_{\mathrm{h}}\right)$. The resonance signal at $1648 \mathrm{~cm}^{-1}\left(v_{\mathrm{C}=\mathrm{O}}\right.$ in amide) and $1562 \mathrm{~cm}^{-1}\left(v_{\mathrm{CN}}\right.$ and $\left.\delta_{\mathrm{NH}}\right)$ are characteristic of amide bonds. The presence of aliphatic chains is attested by vibrational bands at $3000-2800 \mathrm{~cm}^{-1}$ ( $v_{\mathrm{S}}$ and $v_{\mathrm{AS}}$ of $\mathrm{CH}_{2}$ and $v_{\mathrm{S}}$ of $\left.\mathrm{CH}_{3}\right)$ and $1400 \mathrm{~cm}^{-1}\left(\omega_{\mathrm{CH} 2}\right)$. The signal of carbohydrates resonates at $1200-1000 \mathrm{~cm}^{-1}\left(\delta_{\mathrm{CO}}\right)$, where a multitude of bands are partially overlapped and therefore difficult to interpret in detail. The elongation of the $\mathrm{C}=\mathrm{O}$ bonds on acetyl moieties produces an additional band at $1740 \mathrm{~cm}^{-1}$ on spectra of substrates prepared with acetylated $\mathrm{SL}_{\mathrm{a}}$ and $\mathrm{CL}$ 
C1s
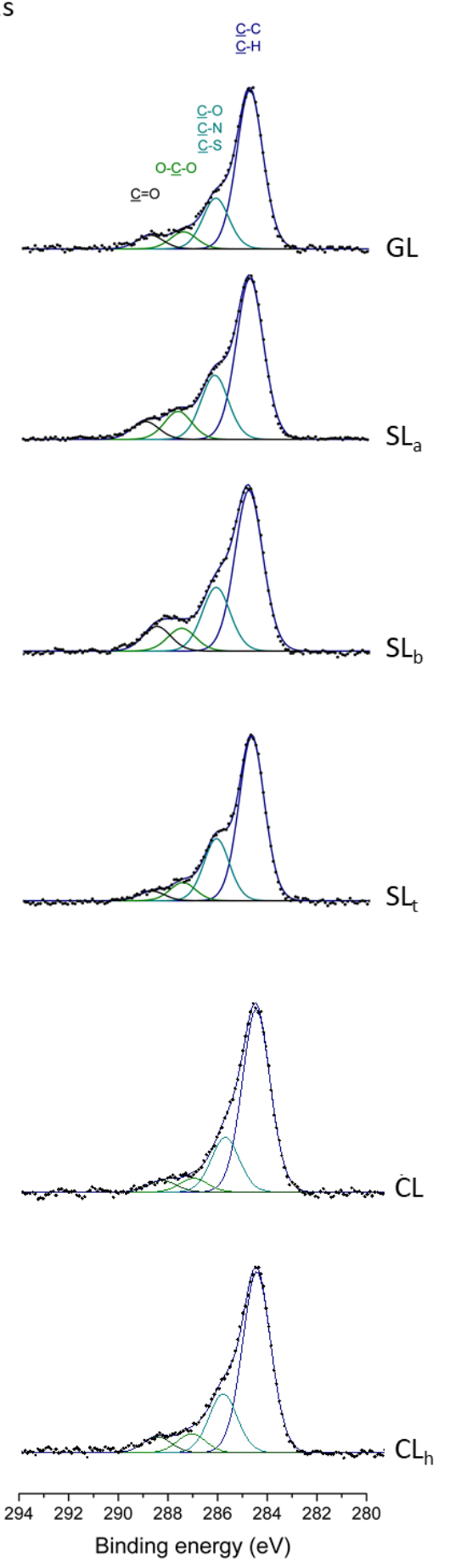

N1s
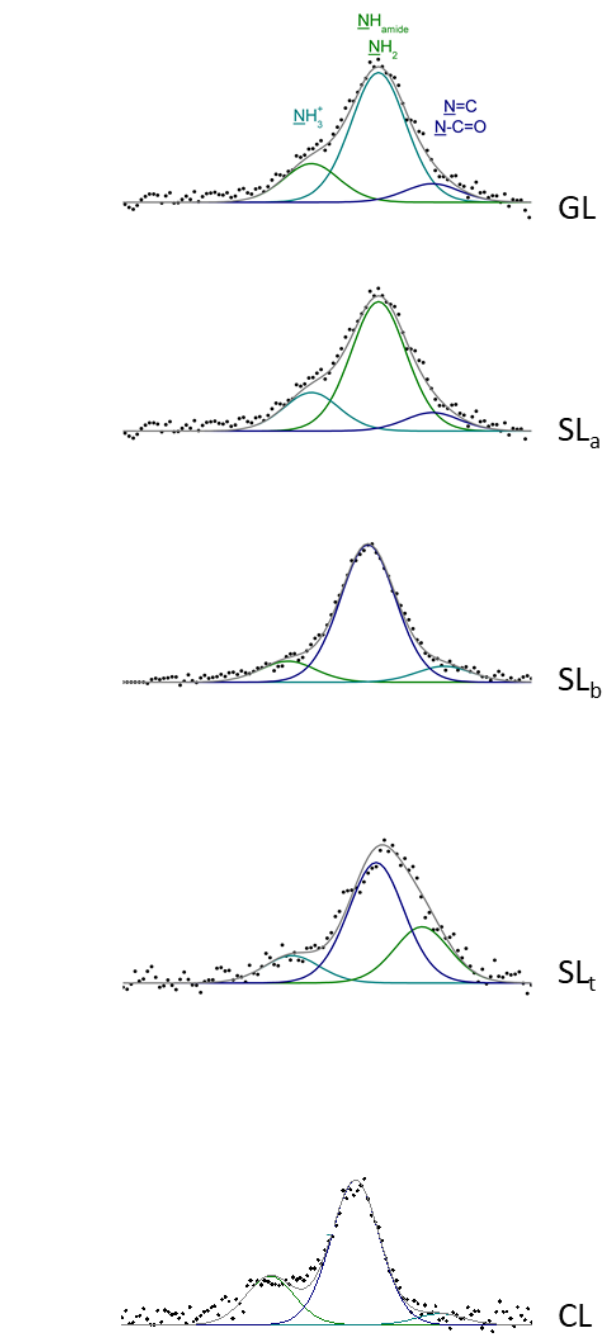

$\mathrm{CL}$

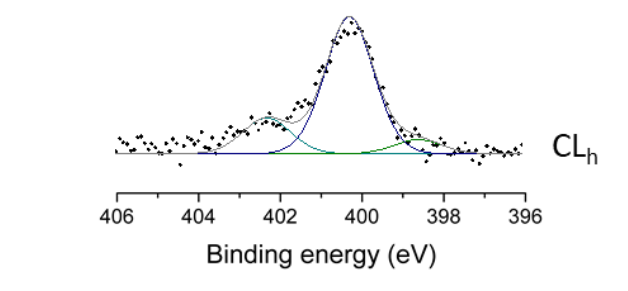

Figure S2. High resolution standardized XPS spectra of the C1s (left) and N1s (right) regions recorded on self-assembled monolayers of glucolipid (GL), cis C18 sophorolipid ( $\mathrm{SL}_{\mathrm{a}}$ ), branched sophorolipid $\left(\mathrm{SL}_{\mathrm{b}}\right)$, trans $\mathrm{C} 18$ sophorolipid $\left(\mathrm{SL}_{\mathrm{t}}\right)$, cellobioselipid $(\mathrm{CL})$ and hydrolyzed cellobioselipid $\left(\mathrm{CL}_{\mathrm{h}}\right)$. The peak at $284.8 \pm 0.1 \mathrm{eV}$ in the $\mathrm{C} 1 \mathrm{~s}$ region attests the presence of an aliphatic chains while the two contributions, at $286.4 \pm 0.1 \mathrm{eV}(\mathrm{C}-\mathrm{OH})$ and $287.7 \pm 0.1 \mathrm{eV}(\mathrm{O}-\mathrm{C}-\mathrm{O})$ are the signature of the carbohydrate groups, also visible in the $\mathrm{O} 1 \mathrm{~s}$. The shift of the nitrogen signal from $401.8 \pm 0.1 \mathrm{eV}\left(\mathrm{NH}_{3}{ }^{+}\right)$on cys primer layer towards $399.9 \pm 0.1 \mathrm{eV}\left(\mathrm{NH}_{2}\right.$ and $\mathrm{NH}$ in amide) after immersion on glycolipids solution demonstrate that the glycolipids are grafted via amide bonds. 


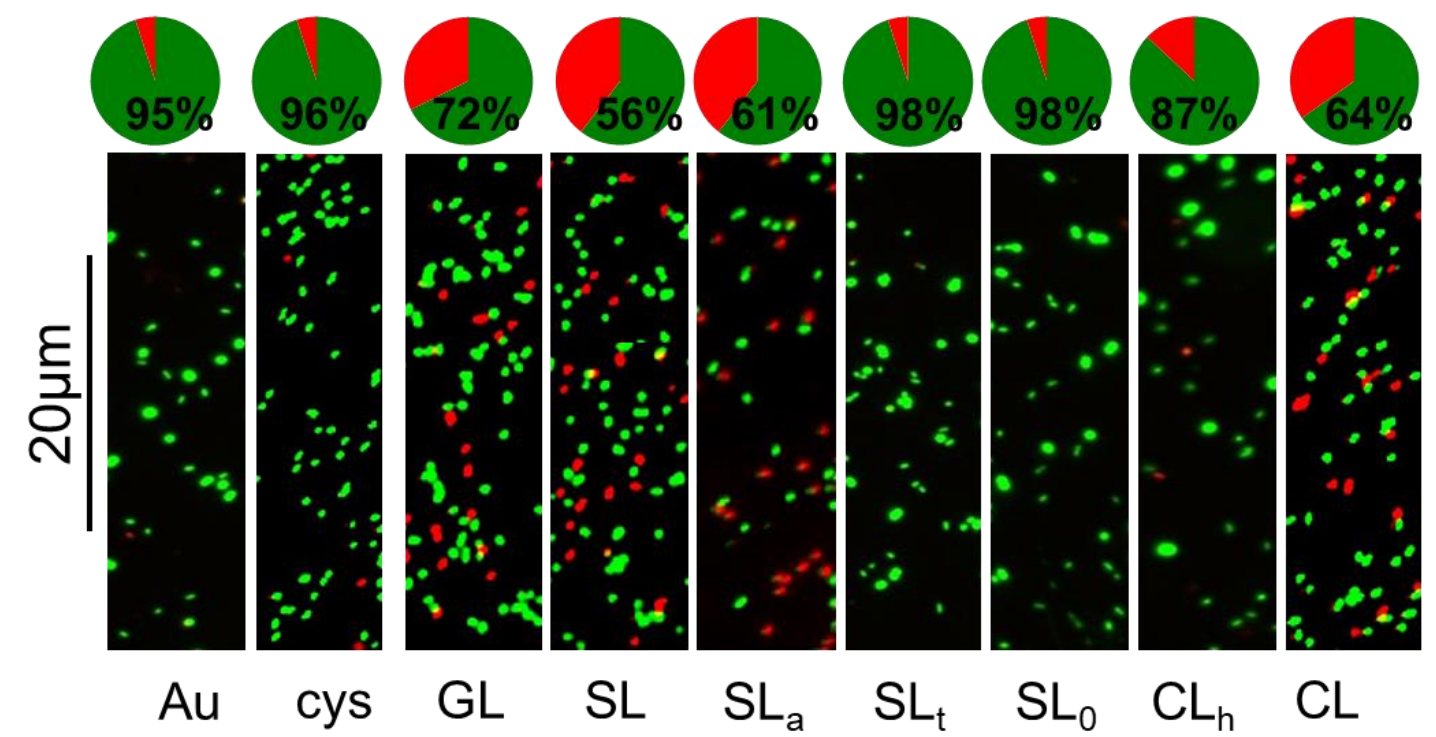

Figure S3. Fluorescent staining evidences membrane damages of bacteria (L. ivanovii) deposited on self-assembled monolayers of glucolipid (GL), deacetylated sophorolipid (SL), cis C18 sophorolipid $\left(\mathrm{SL}_{\mathrm{a}}\right)$, trans $\mathrm{C} 18$ sophorolipid $\left(\mathrm{SL}_{\mathrm{t}}\right)$, saturated sophorolipid $\left(\mathrm{SL}_{0}\right)$, hydrolyzed cellobioselipid $\left(\mathrm{CL}_{\mathrm{h}}\right)$ and cellobioselipid (CL).

Biocompatile gold ( $\mathrm{Au}$ ) surfaces and cysteamine (cys) monolayers constitute negative controls.

The chart above each image represents the proportion of adhering intact (percentage given) and damaged bacteria according to fluorescent staining.

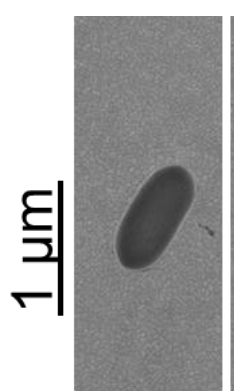

$\mathrm{Au}$

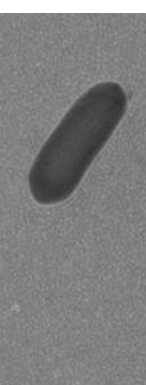

cys

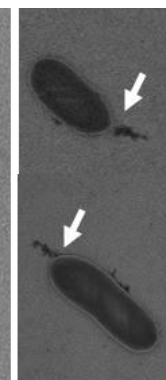

GL

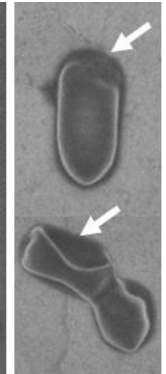

$\mathrm{SL}$

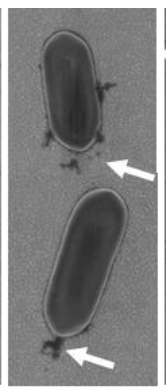

$\mathrm{SL}_{\mathrm{a}}$

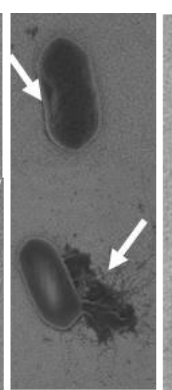

$S L_{t}$

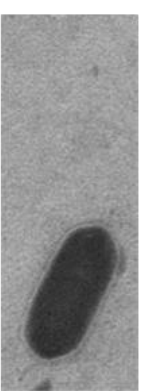

$\mathrm{SL}_{0}$
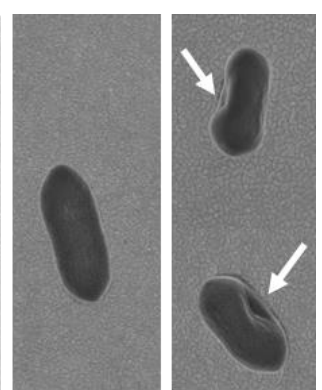

$\mathrm{CL} \quad \mathrm{CL}_{h}$

Figure S4. Scanning electron microscopy reveals qualitative morphological alterations (highlighted by white arrows) of bacteria (L. ivanovii) deposited on self-assembled monolayers of glucolipid (GL), deacetylated sophorolipid (SL), cis C18 sophorolipid $\left(\mathrm{SL}_{\mathrm{a}}\right)$, trans $\mathrm{C} 18$ sophorolipid $\left(\mathrm{SL}_{\mathrm{t}}\right)$, saturated sophorolipid ( $\left.\mathrm{SL}_{0}\right)$, cellobioselipid $(\mathrm{CL})$ and hydrolyzed cellobioselipid $\left(\mathrm{CL}_{\mathrm{h}}\right)$. Biocompatile gold $(\mathrm{Au})$ surfaces and cysteamine (cys) monolayers constitute negative controls. 